\title{
Spinal Cord Stimulation for Treatment of Meralgia Paresthetica
}

\author{
Steven A. Barna, MD, M. Melvin Hu, MD, PhD, Carlos Buxo, MD, Jason Trella, and G. Rees Cosgrove, MD
}

Background: Meralgia paresthetica is a clinical syndrome of pain, dysesthesia or both, in the anterolateral thigh. It is associated with an entrapment mononeuropathy of the lateral femoral cutaneous nerve. Diagnosis of meralgia paresthetica is typically made clinically and is based on the characteristic location of pain or dysesthesia, sensory abnormality on exam, and absence of any other neurological abnormality in the leg. The majority of patients with meralgia paresthetica respond well to conservative treatment.

Objective: To present a case of intractable meralgia paresthetica in which conservative treatment options failed but which was successfully treated with a spinal cord stimulator.

Case Report: A 44-year-old woman presented to the pain clinic with a one-year history of bilateral anterolateral thigh pain. History, physical exam, and diagnostic work-up were consistent with meralgia paresthetica. Multiple medications, physical therapy, and chiropractic therapy were not successful for this patient. In addition, local anesthetic/ steroid injection of the lateral femoral cutaneous nerve provided only short-term relief. Ultimately, a spinal cord stimulator was implanted after a successful temporary percutaneous trial. Two months after the implanta- tion, she continued to have $100 \%$ pain relief, worked full-time, was physically active, and no longer required any pain medication including opioids.

Conclusion: An implanted spinal cord stimulator may be an ideal treatment for intractable meralgia paresthetica after conservative treatments have failed because it is not destructive and can always be explanted without significant permanent adverse effects.

Keywords: Meralgia paresthetica, spinal cord stimulation, spinal cord stimulator, lateral femoral cutaneous nerve, neuropathy
Meralgia paresthetica is a clinical syndrome of pain, dysesthesia or both, in the anterolateral thigh and is associated with an entrapment mononeuropathy of the lateral femoral cutaneous nerve. Typically, the diagnosis of meralgia paresthetica is made clinically, based on the characteristic location of pain or dysesthesia, sensory abnormality on exam, and absence of any other neurological abnormality in the leg. Relief of pain with local anesthetic injection of the lateral femoral cutaneous nerve helps to confirm the diagnosis. The majority of patients with meralgia paresthetica respond well to conservative management alone, although re-

From: MGH Pain Center, Department of Anesthesia and Critical Care, Massachusetts General Hospital Boston, MA, and Department of Neurosurgery, Lahey Clinic, Burlington, MA Address Correspondence:

Steven A. Barna, MD, Medical Director MGH Pain Clinic, Massachusetts General Hospital 15 Parkman Street, WAC 324, Boston, MA 02114 E-Mail: Sbarna@partners.org

Disclaimer: There was no external funding in preparation of this manuscript.

Conflict of Interest: None

Manuscript received on 6/2/2005

Revision submitted on 6/23/2005

Accepted for publication on $6 / 23 / 2005$ current symptoms are not uncommon. On rare occasions surgery is considered for patients with intractable pain that is refractory to conservative treatment. This case report describes the novel use of an implanted spinal cord stimulator to relieve the intractable pain associated with meralgia paresthetica that was refractory to more conservative measures.

\section{Case Report}

A 44-year-old woman was in reasonably good health until one year prior to our initial evaluation. Since she was overweight, she had decided at that time to begin an exercise regimen with a stationary bicycle in an effort to lose weight. Over a period of one month, she gradually built up the intensity and duration of her bicycling. At the end of that month, she noted intense 7-10/10 spontaneous burning dysesthetic pain over bilateral anterolateral thighs. She had no associated weakness or numbness over bilateral lower extremities. She denied any temperature changes, skin color changes, hair growth changes, or nail growth changes in bilateral lower extremities. Bowel and bladder function was intact. She denied abdominal pain, pelvic pain, fever or chills, weight loss, an- orexia, night sweats, vaginal discharge, or gastrointestinal symptoms. Past medical history was significant for asthma, heartburn, smoking, depression, and anxiety. Past surgical history was significant for prior uneventful C-section and abdominal hysterectomy for endometriosis (with resolution of abdominal pain afterwards).

At the time of her initial symptoms, a neurologist and neurosurgeon both consulted on her case. Workup included an electromyogram (EMG), lumbar spine magnetic resonance imaging (MRI), left thigh MRI, and pelvic computed tomography (CT) - all of which were negative. Approximately six months after presentation of her symptoms, the neurosurgeon performed a diagnostic left lateral femoral cutaneous nerve block with $1 \%$ lidocaine. Her pain improved significantly at that time. Subsequently, through the same needle, more lidocaine and methylprednisolone $80 \mathrm{mg}$ were added in hopes of attaining pain relief of longer duration. Unfortunately, the pain relief lasted no more than nine hours.

Prior to coming to our clinic, she had undergone a series of the following treatments without relief of her pain: $5 \%$ lidocaine transdermal patches; gabapentin 
$1200 \mathrm{mg}$ tid; acetaminophen/propoxyphene 650/100; valdecoxib $10 \mathrm{mg}$ a day; topiramate $25 \mathrm{mg}$ bid; acetaminophen/ oxycodone $325 / 10 \mathrm{mg}$ qid; nortriptyline $75 \mathrm{mg}$ a day; ibuprofen, clonazepam, citalopram, rofecoxib, and hydrocodone; physical therapy; and chiropractic therapy.

On physical exam, she was 5'1" tall and 191 pounds, in no apparent distress and with normal vital signs, gait and station. Her back exam revealed full range of motion with flexion, extension and lateral rotation, as well as no tenderness with palpation or spasm. Straight leg raise and Patrick test were negative bilaterally. Abdominal exam was unremarkable. A recent pelvic exam performed by her gynecologist was apparently unremarkable. Her neurologic examination revealed normal bulk and tone, reflexes, and strength of bilateral lower extremities. Sensory examination revealed a decrease in sensation to pinprick and temperature in the anterolateral thighs within the distribution of the lateral femoral cutaneous nerve. No lower extremity allodynia, hyperalgesia, change in skin color, skin temperature, toenail growth, hair distribution, or trophic changes were noted.

At our initial visit, one year after her initial symptoms began, we decided to start her on nortriptyline, which was slowly titrated up to $75 \mathrm{mg}$ per day. She noted some slight improvement in

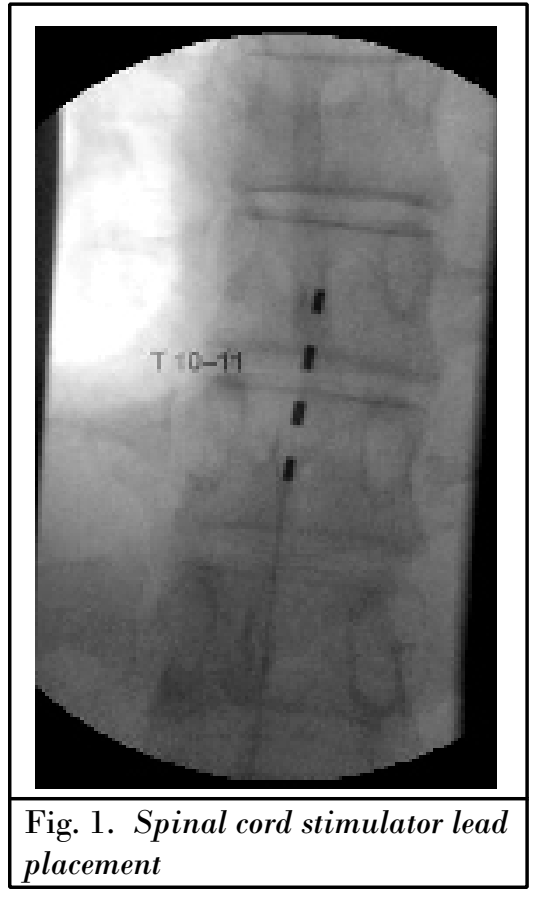

her symptoms but was still in significant pain and required up to five tablets a day of acetaminophen/oxycodone 325/ $10 \mathrm{mg}$. In addition, due to the pain, by this time she was noting increasing difficulty in performing her responsibilities at work as an administrative assistant. Given her poor response to conservative therapy, we discussed the possibility of a spinal cord stimulator implantation. In addition, we discussed the options of surgical transection of the bilateral lateral femoral cutaneous nerves, as previously suggested by the neurosurgeon, and consideration of bilateral peripheral nerve stimulator implantation. Because percutaneous lead spinal cord stimulator trial followed by possible permanent percutaneous lead implantation was felt to be the least invasive of these options, we decided to proceed with the trial. She was referred for consultation to a psychologist (a specialist in chronic pain) to determine whether she would be a good candidate for permanent spinal cord stimulator implantation. She was judged to have no significant psychological factors that would indicate a poor outcome, and we performed a temporary percutaneous spinal cord stimulator trial approximately five weeks after her initial consultation with us.

Under sterile conditions and with fluoroscopic guidance under local anesthesia, a Medtronic Compact Pisces Quad spinal cord stimulator lead was placed percutaneously into the epidural space at L1-L2. The lead tip was then advanced without incident to the midline of the mid-T10 vertebral body level. Stimulation testing at the time revealed excellent coverage of the painful areas. The lead was ultimately secured in place with Steri-Strips ${ }^{\mathrm{TM}}$ and Tegaderm ${ }^{\mathrm{TM}}$ dressing. She was discharged to home with an external pulse generator. At a follow up visit one week later, she noted $100 \%$ satisfaction, had significant reduction of her pain, along with continued excellent coverage of the painful areas. During that period she also stopped both her acetaminophen/ oxycodone 325/10 mg and nortriptyline because she no longer required them for pain relief. The temporary lead was removed without incident.

Approximately one month later she underwent permanent implantation of both the lead and a Medtronic Synergy Versitrel internal pulse generator. Using aseptic technique and under local anesthesia and monitored anesthesia care, an incision was made both along the lumbar spine and the left gluteal area. A Medtronic Pisces Quad spinal cord stimulator lead was placed into the epidural space at L1L2 and advanced to the midline of the mid-T10 vertebral body level (Fig. 1). Stimulation testing at the time revealed excellent coverage of the painful areas. The lead was anchored to the fascia and supraspinous ligament with a plastic anchoring device and 2-0 silk sutures. The internal pulse generator was placed in a subcutaneous pocket made at the left gluteal incision site. The lead and internal pulse generator were connected appropriately with a lead extension and placed percutaneously via a tunneling device between the two incision sites. Both incisions were closed without incident. After observation for a few hours in the recovery unit, she was discharged home.

Eight months after her permanent implantation, she had nearly $100 \%$ pain relief, denied any adverse effects, and was very satisfied with the results. She was working full-time, was more active, was exercising regularly, and was no longer taking any medications for pain. Since her initial presentation to our clinic, she has lost 17 pounds due to diet and consistent exercise.

\section{DISCUSSION}

Meralgia paresthetica is a relatively rare disorder, occurring in 4.3 per 10,000 person years in the general population (1). It is typically associated with obesity with an overlying pannus (2), tight garments around the waist (3), or scar tissue near the lateral aspects of the inguinal ligament. The pain is likely due to some type of injury, compression, or stretching of the lateral femoral cutaneous nerve. It may also occur following a seat belt injury due to a motor vehicle accident, or as the result of injury with iliac bone harvesting during spinal surgery (4). In this case, meralgia paresthetica developed in an overweight woman after she undertook an intensive exercise program of bicycling to lose weight.

Meralgia paresthetica can be difficult to diagnose clinically. It is typically diagnosed based on symptoms and signs in the lateral femoral cutaneous nerve distribution and absence of other neurological abnormality in the lower extremity. Electromyography and nerve conduction studies have a limited role in its diagnosis. Due to variation in anatomy and over- 
lying subcutaneous tissue, stimulation of the lateral femoral cutaneous nerve to record nerve conduction velocity or amplitude is difficult, and frequently requires needle stimulation and recording (5). However, one study showed sensory nerve conduction was the more reliable method for meralgia paresthetica electrodiagnosis than was somatosensory evoked potentials (6). Furthermore, sensory nerve action potential amplitude comparison between the affected and unaffected side in unilateral meralgia paresthetica may be more useful than sensory nerve conduction velocity or somatosensory evoked potential latency comparisons for the diagnosis of meralgia paresthetica (7). More importantly, electrodiagnostic studies may help to rule out other serious underlying neurological abnormalities (5). Temporary relief of pain by injection of local anesthetic around the lateral femoral cutaneous nerve confirms the diagnosis of meralgia paresthetica (8). In this case, injection around the lateral femoral cutaneous nerve with lidocaine and $80 \mathrm{mg}$ methylprednisolone achieved temporary relief of pain and helped confirm the diagnosis. An unremarkable electrodiagnostic study helped to rule out other neurological abnormalities of the lower extremity, but did not aid in diagnosis.

Meralgia paresthetica can be a presenting symptom of a more serious underlying condition. For example, lumbar disc herniation (9), pelvic mass (10), chronic appendicitis (11), and hemangiomatosis (12) have all been reported to present with pain and dysesthesia in the lateral femoral cutaneous nerve distribution typical of meralgia paresthetica. Other potential causes of pain such as complex regional pain syndrome (formerly reflex sympathetic dystrophy), or a peripheral neuropathy due to diabetes, vitamin B deficiency, alcoholism, hypothyroidism, etc., should be investigated. Therefore, a complete workup is necessary to rule out underlying conditions that may present as meralgia paresthetica. In this case, the electrodiagnostic study, lumbosacral spine MRI, left thigh MRI, and CT of the pelvis were all negative. Therefore, more common conditions such as lumbar radiculopathy and pelvic pathology were ruled out.

Meralgia paresthetica typically responds well to conservative treatments such as education to reduce risk factors, avoiding tight garments, and weight loss
(13). Neuropathic pain medications such as gabapentin, and carbamazepine may be helpful to reduce the neuropathic pain and dysesthesia. Williams et al. reported that conservative treatment was successful in relieving symptoms in $91 \%$ of 277 patients with meralgia paresthetica (13) Nerve block with local anesthetic agent and steroid may provide temporary relief of the pain and dysesthesia associated with meralgia paresthetica (14).

For refractory cases, surgical decompression, neurolysis or transection of the lateral femoral cutaneous nerve can provide long-lasting relief for some patients $(4,13,15)$. Nahabedian, et al. reported that 22 out of 23 patients achieved good to excellent outcomes following surgical decompression of the lateral femoral cutaneous nerve (4). Williams et al. reported that 24 patients with intractable symptoms were treated with sectioning of the lateral femoral cutaneous nerve, and 23 patients were successful in symptom relief (13). Van Eerten et al. made a direct comparison between surgical neurolysis and transection in 21 patients with meralgia paresthetica after failure of conservative treatment. Transection was found to be superior to surgical neurolysis for the treatment of meralgia paresthetica (15). Williams et al. proposed that anatomical variations of the nerve and neuromas, which occur frequently, are better treated with transection because neurolysis and transposition may lead to neuroma recurrence (13).

Minimally invasive procedures such as cryoneurolysis or radiofrequency lesioning of the lateral femoral cutaneous nerve are promising, but due to lack of published data their role in managing meralgia paresthetica remains to be seen. Additional treatment options such as peripheral nerve stimulation of the lateral femoral cutaneous nerve may be considered, but experience is lacking. A spinal cord stimulator with a lead anchored to the supraspinous ligament is a less invasive and less destructive option when compared to surgical transection, radiofrequency lesioning, or peripheral nerve stimulator implantation. Furthermore, it may be ultimately explanted with only some minor skin scarring as a final result. In comparison, these destructive options described (with the exception of a peripheral nerve stimulator) carry the risk of actually increasing the pain due to nerve injury or neuroma formation.
In this case, the patient tried neuropathic pain medications including gabapentin (3600 mg per day), topiramate (50 mg per day) and nortriptyline (75 mg per day), which provided only slight relief. She also tried physical therapy, chiropractic therapy, ibuprofen, valdecoxib, rofecoxib, lidocaine transdermal patch, hydrocodone, propoxyphene, and acetaminophen/ oxycodone $325 / 10 \mathrm{mg}$ without significant relief. She also received a local anesthetic with steroid block of the lateral femoral cutaneous nerve, which provided only temporary relief, but did help to confirm the diagnosis (14). Ultimately, the permanent spinal cord stimulator implantation was the least invasive and next logical option when considering more destructive surgical options. Fortunately, she obtained excellent results without any complications to date. In the future, with continued weight loss, the spinal cord stimulator could easily be explanted if her meralgia paresthetica resolves on its own.

Spinal cord stimulation (SCS) is a successful treatment modality for complex regional pain syndrome (16), failed back and neck surgery syndrome, peripheral neuropathic pain such as diabetic neuropathy, ischemic heart disease, and peripheral vascular disease (17). Multiple mechanisms may account for the action of spinal cord stimulation. For example, dorsal horn neuronal activity caused by peripheral noxious stimuli could be inhibited by concomitant stimulation of the dorsal columns (18) Spinal cord stimulation may also modulate the disinhibition of descending analgesia pathways originating in periaqueductal gray matter (19), and induce release of serotonin and substance P in the dorsal horn (20). For treatment outcome, Kemler, et al., in treating complex regional pain syndrome, reported a success rate of $56 \%$ for a group of patients treated with spinal cord stimulation and physical therapy as compared to a group treated with physical therapy alone (16). Turner, et al. systematically reviewed literature related to SCS and failed back surgery syndrome and found an average of $59 \%$ of patients treated with SCS had greater than or equal to $50 \%$ pain relief (21). In addition to the above indications, SCS has also been tried and found to be successful in treating a variety of conditions such as intractable vulvar pain (22), pain associated with thromboangiitis obliterans (Buerger disease) (23), and pain associated with chronic mesenteric 
ischemia (24). In this report, we described meralgia paresthetica as another condition successfully treated by SCS.

\section{ConcLusion}

For meralgia paresthetica refractory to conservative measures including education, medication and local nerve block, long lasting relief in select patients can be achieved by either spinal cord stimulation or surgical options including decompression, neurolysis, or transection of the lateral femoral cutaneous nerve (4, 15). Which option is superior to the other is unclear at this time, and to answer this question requires further clinical studies. Spinal cord stimulation may be superior because it is inherently not destructive, can be performed as a temporary percutaneous trial as a predictor of successful permanent implantation, has no potential to worsen the pain, and can always be explanted without significant permanent adverse effects.

Author Affiliation:

Steven A. Barna, MD

Instructor of Anesthesia

Harvard Medical School

MGH Pain Center

Dept. of Anesthesia and Critical Care

Massachusetts General Hospital

15 Parkman Street, WACC 324

Boston, MA 02114

Email: Sbarna@partners.org

\section{Melvin Hu, MD, PhD}

Clinical Fellow in Pain Medicine

Harvard Medical School

MGH Pain Center

Dept. of Anesthesia and Critical Care

Massachusetts General Hospital

15 Parkman Street, WACC 324

Boston, MA 02114

Carlos Buxo, MD

Clinical Fellow in Pain Medicine
Harvard Medical School

MGH Pain Center

Dept. of Anesthesia and Critical Care

Massachusetts General Hospital

15 Parkman Street, WACC 324

Boston, MA 02114

\section{Jason Trella}

Administrator

MGH Pain Center

Dept. of Anesthesia and Critical Care

Massachusetts General Hospital

15 Parkman Street, WACC 324

Boston, MA 02114

G. Rees Cosgrove, MD

Chief of Neurosurgery

Lahey Clinic

41 Mall Road

Burlington, MA 01805

\section{REFERENCES}

1. van Slobbe AM, Bohnen AM, Bernsen RM, Koes BW, Bierma-Zeinstra SM. Incidence rates and determinants in meralgia paresthetica in general practice. J Neurol 2004; 251:294-297.

2. Deal CL, Canoso JJ. Meralgia paresthetica and large abdomens. Ann Intern Med 1982; 96:787-788.

3. Boyce JR. Meralgia paresthetica and tight trousers. JAMA 1984; 251:1553.

4. Nahabedian MY, Dellon AL. Meralgia paresthetica: etiology, diagnosis, and outcome of surgical decompression. Ann Plast Surg 1995; 35:590-594.

5. Dumitru D, Amato AA, Zwarts MJ. Electrodiagnostic Medicine. Hanley \& Belfus, Philadelphia, 2002, pp 865-866.

6. Seror P. Lateral femoral cutaneous nerve conduction versus somatosensory evoked potentials for electrodiagnosis of meralgia paresthetica. Am J Phys Med Rehabil 1999; 78:313-316.

7. Lagueny A, Deliac MM, Deliac P, Durandeau A. Diagnostic and prognostic value of electrophysiologic tests in meralgia paresthetica. Muscle Nerve 1991; 14:51-56.

8. Ivins GK. Meralgia paresthetica, the elusive diagnosis: clinical experience with 14 adult patients. Ann Surg 2000; 232:281286.

9. Trummer M, Flaschka G, Unger F, Eustacchio S. Lumbar disc herniation mimicking meralgia paresthetica: case report. Surg
Neurol 2000; 54:80-81.

10. Suber DA, Massey EW. Pelvic mass presenting as meralgia paresthetica. Obstet Gynecol 1979; 53:257-258.

11. Ghavanini MR, Ghavanini AA. Meralgia paresthetica as the presenting feature of chronic appendicitis. Am J Phys Med Rehabil 2001; 80:703-705.

12. Yamamoto T, Kurosaka M, Marui T, Mizuno $\mathrm{K}$. Hemangiomatosis presenting as meralgia paresthetica. J Pediatr Surg 2001; 36: 518-520.

13. Williams PH, Trzil KP. Management of meralgia paresthetica. J Neurosurg 1991; 74: 76-80.

14. Grossman MG, Ducey SA, Nadler SS, Levy AS. Meralgia paresthetica: diagnosis and treatment. J Am Acad Orthop Surg 2001; 9: 336-344.

15. Van Eerten PV, Polder TW, Broere CA. Operative treatment of meralgia paresthetica: transection vs neurolysis. Neurosurgery 1995; 37:63-65.

16. Kemler MA, Barendse GA, van Kleef $M$, de Vet HC, Rijks CP, Furnee CA, van den Wildenberg FA. Spinal cord stimulation in patients with chronic reflex sympathetic dystrophy. N Engl J Med 2000; 343:618-624.

17. Stojanovic MP. Stimulation methods for neuropathic pain control. Current Pain and Headache Reports 2001; 5:130-137.

18. Dubuisson D. Effect of dorsal column stimulation on gelatinosa and marginal neu- rons of cat spinal cord. J Neurosurg 1989 70:257-265.

19. Stiller CO, Linderoth B, O'Connor WT, Franck J, Falkenberg T, Ungerstedt U, Brodin E. Repeated spinal cord stimulation decreases the extracellular level of gamma-aminobutiric acid in periaqueductal grey matter of freely moving rats. Brain Res 1995; 699: 231-241.

20. Linderoth B, Gazelius B, Franck J, Brodin E. Dorsal column stimulation induces release of serotonin and substance $P$ in the cat dorsal horn. Neurosurgery 1992; 31: 289-296.

21. Turner JA, Loeser JD, Bell KG. Spinal cord stimulation for chronic low back pain: a systematic literature synthesis. Neurosurgery 1995; 37:1088-1096.

22. Whiteside JL, Walters MD, Mekhail N. Spinal cord stimulation for intractable vulvar pain. A case report. J Reprod Med 2004; 48:821-823.

23. Manfredini R, Boari B, Gallerani M, la Cecilia O, Salmi R, de Toma D, Galeotti R, Ragazzi R. Thromboangiitis obliterans (Buerger disease) in a female mild smoker treated with spinal cord stimulation. Am J Med Sci 2004; 327:365-368.

24. Ceballos A, Cabezudo L, Bovaira M, Fenollosa P, Moro B. Spinal cord stimulation: a possible therapeutic alternative for chronic mesenteric ischemia. Pain 2000; 87:99101. 